\title{
SoC it to EM: electromagnetic side-channel attacks on a complex system-on-chip
}

\author{
J. Longo ${ }^{1}$, E. De Mulder ${ }^{2}$, D. Page ${ }^{1}$, and M. Tunstall ${ }^{2}$ \\ 1 Department of Computer Science, University of Bristol, \\ Merchant Venturers Building, Woodland Road, \\ Bristol, BS8 1UB, United Kingdom. \\ \{jake.longo, daniel.page\}@bristol.ac.uk \\ 2 Rambus Cryptography Research Division, \\ 425 Market Street, 11th Floor, \\ San Francisco, CA 94105, United States. \\ \{elke.demulder, michael.tunstall\}@cryptography.com
}

\begin{abstract}
Increased complexity in modern embedded systems has presented various important challenges with regard to side-channel attacks. In particular, it is common to deploy SoC-based target devices with high clock frequencies in security-critical scenarios; understanding how such features align with techniques more often deployed against simpler devices is vital from both destructive (i.e., attack) and constructive (i.e., evaluation and/or countermeasure) perspectives. In this paper, we investigate electromagnetic-based leakage from three different means of executing cryptographic workloads (including the general purpose ARM core, an on-chip co-processor, and the NEON core) on the AM335x SoC. Our conclusion is that addressing challenges of the type above $i s$ feasible, and that key recovery attacks can be conducted with modest resources.
\end{abstract}

Keywords: side-channel, electromagnetic, system on chip, ARM, NEON.

\section{Introduction}

A significant proportion of academic literature on side-channel attacks already targets real-world devices: even a very limited list of examples, such as those against KeeLoq keyless entry systems [20]28, Xilinx FPGA bit-stream encryption [34, or Atmel CryptoMemory [5] authentication, provides compelling evidence of their potency. However, and although clear counter-examples are identifiable, such devices may often be characterised as electronically and/or architecturally simple (the cryptographic aspects at least). From one perspective, this is a non-issue: use-cases such as contact-based and contactless payment cards, and trends such as Internet of Things (IoT) suggest devices of this type will abound for some time to come; the simplicity of a target in no way implies that developing and mounting attacks is simple nor without more general value. However, from a different perspective, it may seem unsatisfactory since it contrasts sharply with trends in commodity micro-electronics. In particular, more complex devices with richer functionality are now routinely deployed in contexts where 
side-channel attacks are a threat. For example, smart-phones now house multicore, System-on-Chip (SoC) components with multi-gigahertz clock frequencies as standard, and, modulo constraints such as energy efficiency, market forces will drive increased use of similar components over time.

Within this context, use of ElectroMagnetic (EM) side-channel leakage is particularly attractive. Rohatgi [38] offers a comprehensive overview of both the physical phenomenon itself plus seminal results such as [3|21|37, neither of which we expand on unless specifically relevant. Versus power analysis 29], the non-contact, spatially flexible nature of an EM-based alternative means it a) represents a less invasive means of taking acquisitions, b) avoids issues such as on-chip voltage regulation and, most importantly, c) permits targeting of specific regions of (or components on) an SoC that otherwise offer composite leakage.

Our goal in this paper is to demonstrate that by carefully translating and refining existing techniques, EM-based side-channel attacks are viable against modern, complex targets. The challenges of evaluation and countermeasure instrumentation already motivate such work, but are arguably magnified by other constructive applications of side-channels (e.g., protection of intellectual property [7] and Trojan hardware detection [19]) relevant to SoCs: all benefit from better, open (noting this topic seems to represent an active but largely undocumented focus of various security services [40]) understanding of the associated leakage characteristics. We explore a single exemplar target device, namely the Texas Instruments (TI) AM335x SoC on a BeagleBone Black development board, with respect to three options for execution of cryptographic workloads. Following the relevant background material in Section 2, our contribution is, concretely, the EM-based analysis of

1. AES executed by an OpenSSL server on the ARM core (Section 3),

2. the proprietary AES co-processor (Section 4), and

3. the NEON ${ }^{3}$ core, including bit-sliced AES (Section 5).

A central conclusion is that, while some effort is required to characterise the leakage, attack complexity does not necessarily scale in line with perceived device complexity. For example, in the first case above we are able to acquire and exploit leakage at much lower frequencies than suggested by the $1 \mathrm{GHz}$ system clock; this implies attack cost may also be lower than expected, and hence relying on device complexity (resp. obscurity) to provide security is dubious at best.

\section{Background}

\subsection{An overview of the BeagleBone platform}

BeagleBone Black is a single-board computer built around a AM335x "Sitara" SoC. Constituent components can be grouped logically into four sub-systems

\footnotetext{
${ }^{3}$ Note that by targeting NEON, we specifically aim to add detail to the premise introduced during the CHES 2014 rump session talk of Bernstein and Lange: see http://cr.yp.to/talks/2014.09.25-2/slides-dan+tanja-20140925-2-4x3.pdf
} 


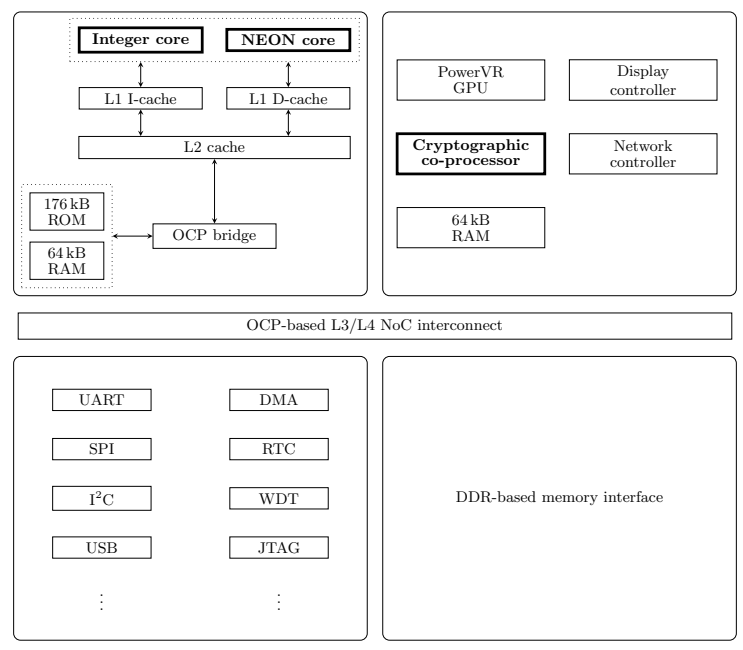

Fig. 1: A block diagram of the AM335x SoC, replicating [26, Figure 1-1]; note that components and sub-systems pertinent to sections in the paper are highlighted.

per [26, Figure 1-1] (which is reproduced in Figure 1 for the sake of completeness); the sub-systems are able to communicate via a dedicated Network-on-Chip (NoC), or interconnect. The following sections focus on the Micro-Processor Unit (MPU) and the cryptographic co-processor. Although the latter lacks public documentation of the internal design (bar device driver source code ${ }^{4}$ that interfaces with it), the former warrants further analysis: we refer to the extensive literatur $^{5}$ for in-depth coverage. In short, however, alongside L1 and L2 caches and on-chip memory, the central 32-bit ARM Cortex-A8 MPU core is clocked up to $1 \mathrm{GHz}$. As they dominate cryptographic use-cases, we focus on the execution of integer instructions by this core, which has two distinct pipelines: the 13-stage ARM pipeline (or core) and 10-stage NEON pipeline (or core), essentially a SIMD co-processor marketed as an accelerator for multimedia and signal processing workloads. Within the ARM pipeline, the 6 execution stages are supported by two symmetric integer pipelines, a multiplier pipeline and a load/store pipeline; operands are supplied from a 16-entry register file of 32-bit registers. Within the NEON pipeline, the 6 execution stages are supported by integer ALU, Multiply-ACcumulate (MAC) and load-store pipelines; operands are supplied from a 256-byte register file, which can be viewed as comprising 32 double-word (64-bit) or 16 quad-word (128-bit) entries (each with sub-words of $w \in\{8,16,32,64,128\}$ bits) depending on the instruction type. Both pipelines are dual-issue (meaning 2 instructions can be issued per cycle, modulo dependencies), and in-order (meaning instructions complete in the same order they were issued). Instructions enter the NEON pipeline after having been decoded by the ARM pipeline, with the two decoupled using a 16-entry queue.

4 http://github.com/torvalds/linux/blob/master/drivers/crypto/omap-aes.c

5 http://www.arm.com/files/pdf/A8_Paper.pdf 
The central point to take away from such analysis is the high degree of architectural complexity evident, even ignoring the number of components. For example, the MPU alone has a total of 3 clock and 4 power domains. Such features make the $\mathrm{SoC}$ an extremely challenging target with respect to Signal-toNoise Ratio (SNR), and underlines the advantages offered by EM-based leakage.

\subsection{Experimental environment}

Acquisition and measurement equipment. To allow reproducibility, the equipment used throughout this paper is listed below:

- Tektronix DPO7104 $1 \mathrm{GHz}$ oscilloscope,

- Signatec PX14400 $400 \mathrm{MS} / \mathrm{s}$ digitiser,

- Langer PA303 pre-amplifier plus various (e.g., low-pass) hardware filters,

- Langer RF-3 mini near-field probe set,

- Langer ICS105 IC scanner (or XY-table),

- Matlab 2014b (with signal processing toolbox).

The configuration was therefore very standard: the target device was mounted on the XY-table to allow micro-positioning of the probe(s), which supplied an amplified, filtered signal to either the digitiser (Section 3 and Section 5) or oscilloscope (Section 4 to cope with a higher sampling rate). This limits our remit strictly to close-range acquisitions, rather than at a distance, e.g., per [43].

Software stack. We used a standard BeagleBone Black distribution of Debian "Wheezy" on the target device (Linux kernel version 3.13.3). The device was booted from on-board embedded MultiMediaCard (eMMC) storage as is; no standard system processes were disabled. On top of this platform we use OpenSSL 1.0.1 $j$, with the cryptodev extension ${ }^{6}$ enabled when appropriate.

\subsection{Leakage detection and exploitation strategy}

Notation. For some set or sequence $x$ of length $n$, we let $x[j]$ denote the $j$-th element of $x$ such that $0 \leq j<n ; x_{i}$ then denotes the $i$-th such object within a larger collection, with the subscript omitted where irrelevant. We use $\mathcal{H}(x)$ and $\mathcal{D}(x, y)$ to denote the Hamming weight and distance, respectively, of some $x$ and $y$. As such,

$$
r_{i}=\operatorname{DUT}_{k}^{f}\left(x_{i}\right) \rightsquigarrow \lambda_{i}
$$

models some $i$-th execution of an operation $f$ on the target device DUT, involving a security critical datum $k$ (e.g., key material), accepting input $x_{i}$ and yielding an output $r_{i}$ and an EM-based trace of leakage $\lambda_{i}$ (a sequence of samples). Depending on the context, the target operation ranges from single instructions to entire algorithms (e.g., AES), and, from the attacker perspective, $r_{i}$ and/or $x_{i}$ may be known or unknown and controlled or uncontrolled.

\footnotetext{
6 http://cryptodev-linux.org/
} 
$\lambda$ is essentially a function of the target device (or leakage model), the operation $f$ and input $x_{i}$, plus the probe type and location (and any other parameters of the experimental environment). With this in mind, mounting a concrete attack demands an attacker a) determines when and where (in time and/or frequency domains) $\lambda$ contains useful, exploitable leakage, and b) selects a probe configuration to maximise said leakage (i.e., maximise the SNR).

Leakage detection. While several strategies, e.g., [11|12|23, have been proposed to address the former challenge above, throughout this paper we use Welch's t-test. More specifically, we use the Test Vector Leakage Assessment (TVLA) methodology of Goodwill et al. 23. Although there are several varients (fixedversus-random and semi-fixed-versus-random for instance), the basic idea involves constructing two sets of test vectors $V_{0}$ and $V_{1}$ : the former contains a single (semi-)fixed vector, whereas the latter contains (a large number of) vectors chosen uniformly at random. Note that Uno et al. [42, Section II.A] use a special-case of this approach, with fixed and random test vectors replaced by full- and zero-weight alternatives (the assumed maximum and minimum cases of leakage under a Hamming weight model). For each $i$-th invocation of the target device, the input is selected by first randomly selecting a test vector type, i.e., a $b \stackrel{\$}{\leftarrow}\{0,1\}$, then a test vector from the appropriate set, i.e., an $x_{i} \stackrel{\$}{\leftarrow} V_{b}$; the resulting trace of leakage, $\lambda_{i}$, is added to a set $\Lambda_{b}$ based on the test vector type. Then, we compute the t-statistic trace as

$$
t=\frac{\bar{\Lambda}_{0}-\bar{\Lambda}_{1}}{\sqrt{\frac{\sigma_{0}^{2}}{\left|\Lambda_{0}\right|}+\frac{\sigma_{1}^{2}}{\left|\Lambda_{1}\right|}}}
$$

where $\left|\Lambda_{b}\right|, \bar{\Lambda}_{b}$ and $\sigma_{b}^{2}$ respectively denote the sample size, sample mean and sample variance of set $\Lambda_{b}$. The idea is that given a threshold $\tau$ (say $\tau=4.5$ per [23, Section 3]), if we find $|t[j]|>\tau$ then we claim significant leakage is detected at the $j$-th sample: at that point there is a statistically observable difference between fixed and random test vectors, so there may be data-dependent and thus potentially exploitable information present. Each following section uses this approach: section-specific detail is included where appropriate, with a comprehensive overview deferred to Appendix A.

\section{Software-based AES}

In the literature, it is common to target embedded devices (e.g., a micro-controller) executing a program on "bare-metal", i.e., directly on the hardware. Although reasonable for some scenarios, a growing number of targets will execute an Operating System (OS) kernel; this is even true of many smart-cards (cf. JavaCard or MULTOS). Perhaps due to the perceived increase in complexity, related attacks are less common than the bare-metal case: selected examples include Uno et al. 42] and Genkin et al. 22] who mount non-differential EM-based attacks on RSA (plus ElGamal in the latter case only) executing under Android (on ARM) 

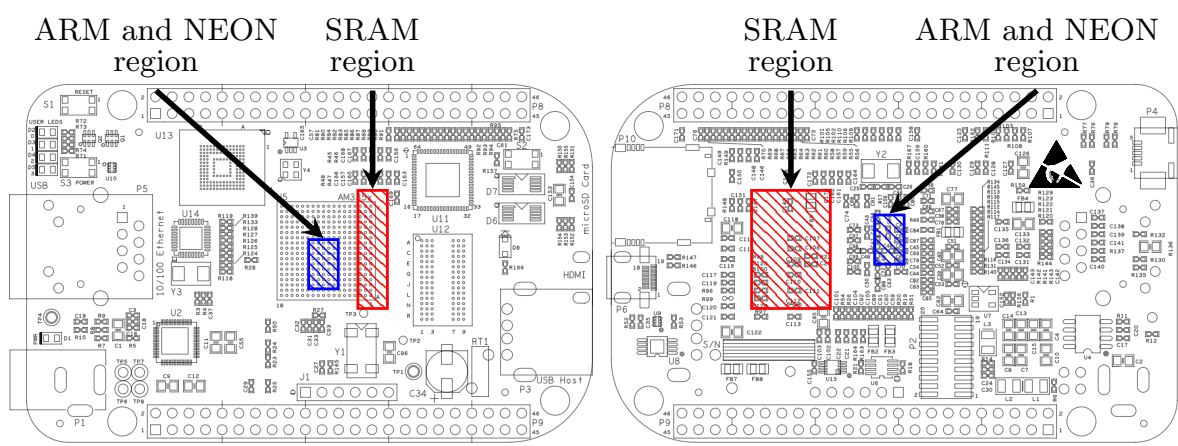

Fig. 2: BeagleBone Black schematic (source: http://github.com/CircuitCo/ BeagleBone-Black/blob/rev_a5c/BBB_PCB.zip) from front-side (left) and back-side (right), annotated with probe locations for leakage from the SRAM (red) and ARM and NEON cores (blue).

and Windows XP (on x86), Aboulkassimi et al. 112 who mount differential EM-based attacks on AES executing under Java ME (on ARM), and Pellegrini et al. 35] who mount voltage depletion fault attacks on RSA executing under Linux (on SPARC).

In this section we consider a systems-oriented scenario of the latter type. Specifically, we imagine the target is a communications device (e.g., a smartphone) engaged in a TLS-based session with some server. As such, the attacker can observe computation of

$$
c_{i}=\mathrm{DUT}_{k}^{\mathrm{AES}-128-\mathrm{CBC}}\left(m_{i}\right) \rightsquigarrow \lambda_{i} .
$$

That is, AES-128 encryption, in CBC mode, of some unknown plaintext $m_{i}$ under $k$ to yield a known ciphertext $c_{i}$ (since it is communicated across the network). Concretely, each encryption operation is performed by OpenSSL in software via the default T-tables-based [14, Section 4.2] implementation.

\subsection{Experimental outline}

Before considering an attack strategy to exploit $\lambda$ and recover $k$, a host of experimental challenges need to be addressed: the first relates to acquisition of $\lambda$. In common with analysis of other unknown/as yet unprofiled target devices, we rely on initial exploration based on full control of a replica profiling device. We stress that our use of the profiling device is simply to give insight into the associated signal characteristics: although it is well known that such an approach is not necessarily sufficient for building templates [13, we simply use it to mitigate systemic features (e.g., of the OS scheduler, hardware and software interrupts etc.) inherent in the scenario above.

Probe location. An initial, manual scan of the SoC surface was conducted in several stages to identify leakage related to the execution of three kernels: a set of memory intensive operations, a spin-lock, and a set of computationally 


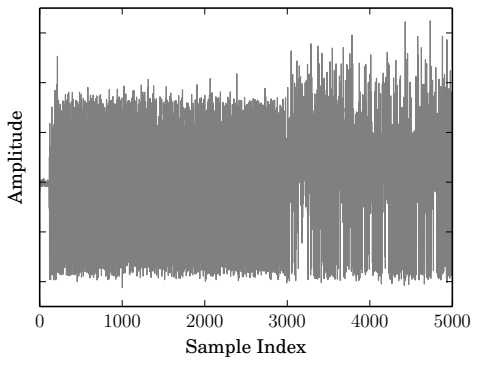

(a) Uninterrupted.

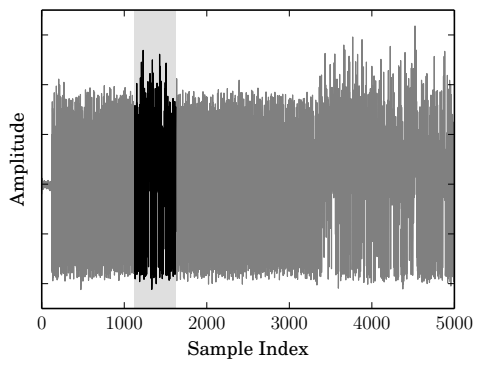

(b) Interrupted.

Fig. 3: Impact of interrupts on the acquisition process in Section 3 whereas the uninterrupted case (left) yields a "clean" trace, the interrupted case (right) is corrupted (during the annotated period).

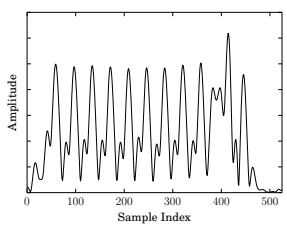

(a) $300 \mathrm{MHz}$.

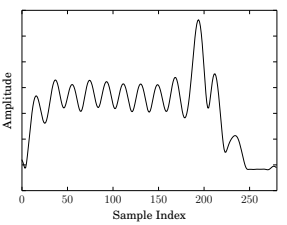

(b) $600 \mathrm{MHz}$.

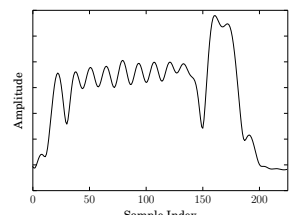

(c) $800 \mathrm{MHz}$.

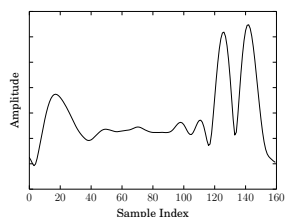

(d) $1 \mathrm{GHz}$.

Fig. 4: Impact of clock scaling on the acquisition process in Section 3 , each trace represents execution of AES under one of the four available clock frequencies.

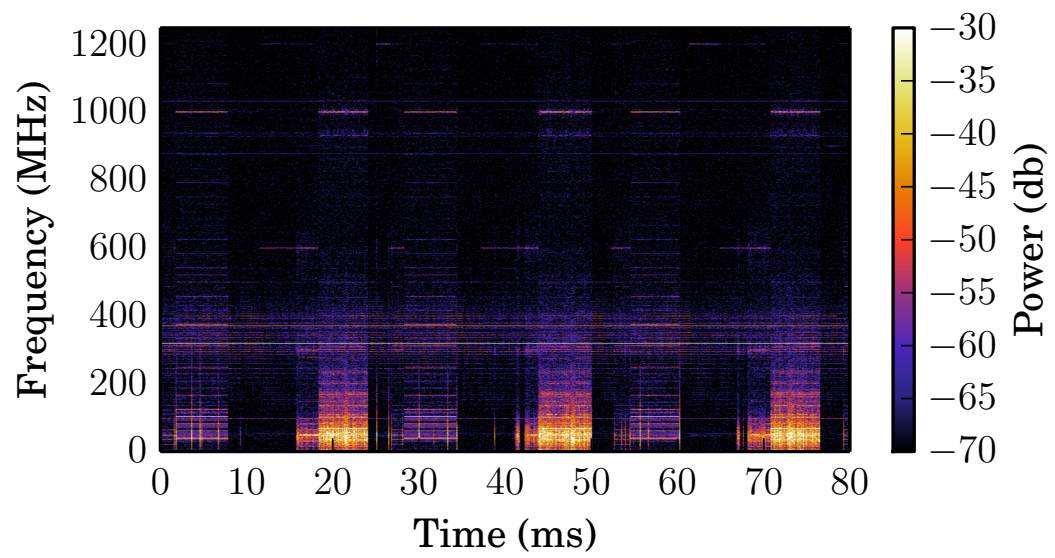

Fig. 5: Spectrogram plot of frequency band 0 to $1.25 \mathrm{GHz}$ at our attack location, with the profiling device cycling through three kernels: a total of three iterations (left-to-right) of the memory intensive, spin-lock and computational (i.e., AES) kernels is illustrated. Note a) the indicative frequency response of AES, and b) the relatively narrow, low frequency range required to capture this response. 


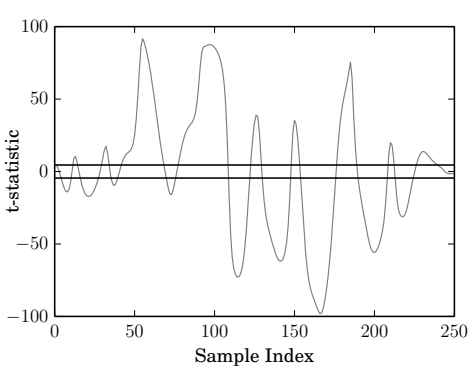

(a) Fixed-versus-random test.

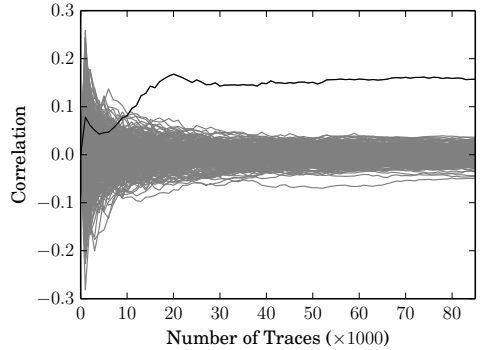

(b) Single-bit correlation.

Fig. 6: Leakage analysis results for a free-running target using 85,000 sub-traces matched at $600 \mathrm{MHz}$. The leakage detection test (left) shows leakage above the significance threshold $\tau=4.5$ (marked in black on the Y-axis) throughout the AES execution. The single-bit analysis (right) tracks the correct key bit hypothesis of the first byte over the number of sub-traces used.

intensive operations (namely AES encryption, as performed by OpenSSL). By periodically cycling through the kernels and monitoring the frequency response (illustrated by Figure 5), two distinct regions of interest were identified (shown in Figure 2). We attribute one region (straddling the SoC edge and SRAM bus) to the memory intensive kernel, and hence memory access. Given that the memory bus is specific to this development kit, that no effort appears to have been made to secure it, and that a complete operational datasheet is availabl ${ }^{7}$. we did not feel it warranted further investigation even though it will likely yield exploitable leakage. The second region is located centrally on the AM335x surface, over a cluster of capacitors on the back-side of the board (specifically, around C94 and C46). Motivated by a) the magnitude of the frequency response observed during the AES execution, but also b) the long-term trend toward stacked fabrication processes (and associated decrease in SNR), we fixed the probe location over this support circuitry.

Acquisition tuning. The leakage from the selected region was identifiable using relatively low frequencies alone, i.e., below $100 \mathrm{MHz}$. We expect this, to some degree, since a) AES throughout is clearly lower than the system clock frequency (one round is requires more than one instruction), and b) the on-chip discrete components coupled with the capacitor behave as a low-pass filter. As such, we applied a band-pass filter to the amplified signal centred on $45 \mathrm{MHz}$ with a bandwidth of $24 \mathrm{MHz}$. This is interesting in so far as it allows use of less capable, and hence less expensive acquisition equipment. That is, rather than use a high-specification oscilloscope, we were able to use a lower-specification digitiser.

Bulk, or multi-block acquisitions. Our next step was to replace the artificial kernels with a "free-running" uninstrumented OpenSSL client instance: no artificial

\footnotetext{
7 http://www .micron.com/parts/dram/ddr3-sdram/mt41k256m16ha-125
} 
(e.g., hardware, via a GPIO pin) triggers are inserted. A coarse, soft trigger is attractive, but strictly to limit acquisitions to a target session (i.e., one $k$ ) rather than to provide alignment per se: each trace $\lambda_{i}$ acquired therefore relates to $l$ invocations of AES rather than 1, i.e., each $c_{i}$ is now a $(16 \cdot l)$-byte or $l$-block ciphertext, as generated by the TLS record layer. Such a soft trigger is easily realised via traffic analysis, and is additionally beneficial since it permits $c_{i}$ to be known (i.e., sniffed) rather than controlled (i.e., injected) by the attacker.

Although we argue that this choice is more realistic than the alternative, it has both positive and negative effects. On one hand, bulk acquisition significantly reduces the wall-clock time required [6, Section 3] and allows the effect of data and instruction caches to be largely ignored (since all but the first few AES invocations will occur with a warm cache). On the other, we must address various challenges relating to systematic noise that occurs over the longer time period (and which for "one-shot" devices such as smart-cards, are normally irrelevant).

Interrupt detection and synchronisation. The OS may preempt a user process if it does not voluntarily yield, or if the kernel needs to service an interrupt: use of bulk acquisition necessitates we account for these interrupts, because they will be observed more frequently during the computation of longer ciphertexts.

Figure 3 demonstrates that an uninterrupted versus interrupted trace can be identified visually. We automate similar identification using the trace alignment scores (i.e., a measure of the least squares [31, page 208]). To do so, we manually identify and select a single uninterrupted trace for use as a template. We then perform coarse alignment of all traces and record their score (versus the template): if the score is above an experimentally determined threshold, the trace is assumed to have been interrupted. For interrupted traces, we then have two choices: discard them, or "clean" them by pruning the interrupted region. We found the low sample rate used means interrupt pruning is highly error prone (it is not always clear at which exact point the interrupt has ended and the OpenSSL process has resumed); such errors desynchronise the trace (i.e., the sub-trace for a given AES invocation) from the associated ciphertexts. Although discarding traces imposes a penalty on the total number of traces required, we opted for this approach.

Clock scaling. Finally, the OS may attempt to scale (or throttle) the clock frequency to optimise power consumption. We observed cases where this occurred, although found the device would typically stabilise at $600 \mathrm{MHz}$ once the OpenSSL process becomes active. AES execution under each clock configuration is shown in Figure 4, with the difference between cases clearly highlighting the resulting misalignment.

By sampling well above the Nyquist rate, it may be possible to infer the clock rate by examining the response at specific frequencies (i.e., at $300 \mathrm{MHz}$, $600 \mathrm{MHz}, 800 \mathrm{MHz}$ and $1 \mathrm{GHz}$ ); such an approach may also facilitate interrupt pruning (per the above). However, use of a low sampling rate, while advantageous in other respects, rules this approach out. Instead, we simply created a template of AES execution at each clock frequency: any trivial comparison between each 
template and target trace reveals the clock frequency used, and yields a usable subset of traces whose clock frequency is uniform.

\subsection{Analysis and discussion}

Summarising the section above, to mount a concrete attack we performed an acquisition phase as follows:

1. Bulk acquire $n=1,000$ traces, each including $l=256$ encryption operations (meaning $4 \mathrm{kB}$ of traffic per trace, and $\sim 4 \mathrm{MB}$ in total).

2. Deal with systemic noise by filtering for interrupts and clock scaling; in our experience, this means discarding $\sim 20 \%$.

3. From each remaining trace, extract a fragment or sub-trace for each encryption operation; match these with the associated ciphertexts.

4. Realign each sub-trace, and discard any corrupted or low-quality cases; in our experience, this means discarding a further $\sim 5 \%$.

This process yields a set of $s<n \cdot l$ remaining (sub-)traces, and for such $n$ and $l$ took $\sim 6 \mathrm{~min}$. Based on this set, we then attempted to exploit the leakage. To do so, we mounted a single-bit correlation-based attack targeting the T-table (or S-box) look-up in the final AES round. Figure 6billustrates, without loss of generality, an example for the first byte of $k$ : it shows growth of the correlation coefficient as the number of (sub-)traces increases. Note the correct (highlighted) hypothesis is clearly distinguished using around 20,000 (sub-)traces, meaning we could have bulk acquired as few as 100 traces $(\sim 400 \mathrm{kB})$ and still have been successful. In reality, fine-tuning $n$ before the acquisition phase is difficult since the attacker cannot control $l$ (and indeed this may change from one trace to another). However, using an adaptive choice of $n$ such that $s$ is large enough, the attack still succeeds.

We benchmarked this attack against a traditional alternative where an artificial hardware trigger (which simultaneously aligns traces, and avoids the issue of interrupts) and fixed clock frequency were used. We found key recovery was possible with only 3,000 (sub-)traces, $\sim 7$ times fewer than our free-running scenario. We posit the gap between the two can be incrementally reduced, since it essentially represents pre-processing inefficiency, deferring this to future work.

\section{Hardware-based AES}

In this section, we shift focus to hardware-based execution of AES using the cryptographic co-processor. As in Section 3 the attacker can still observe the computation of

$$
c_{i}=\mathrm{DUT}_{k}^{\mathrm{AES}-128-\mathrm{CBC}}\left(m_{i}\right) \rightsquigarrow \lambda_{i}
$$

as invoked via OpenSSL. However, the underlying encryption operations are performed using a hardware-based AES implementation. We suggest this is likely to reflect use-cases such as Full Disk Encryption (FDE) given the potential to marshal operations via DMA. In such a use-case, the attacker can access $c_{i}$ since this will represent (a block of) ciphertext stored on, and readable from, the disk in question. 


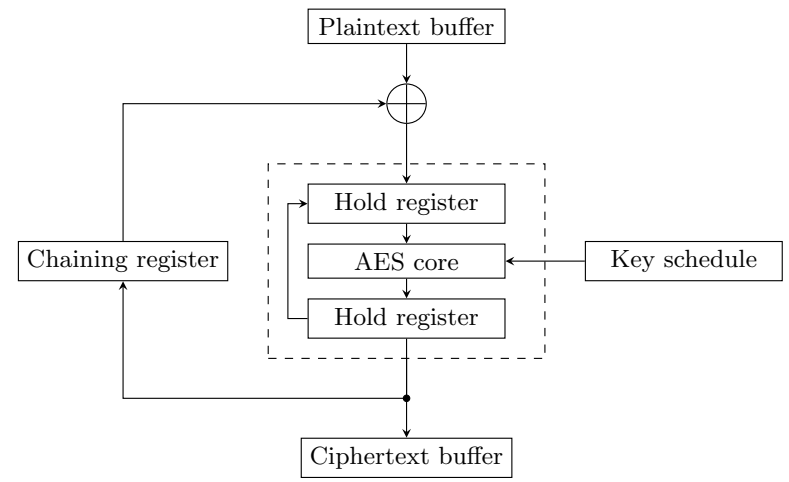

Fig. 7: Probable high-level architecture for the AES core within the AM335x cryptographic co-processor (CBC mode): note that various registers may be merged (e.g., input and output hold registers), and the chaining register should be initialised with an IV.

\subsection{Experimental outline}

As noted in Section 2.1, there is scant documentation for the AM335x cryptographic co-processor: our only insight into the internal design stems from device drivers that support interaction with it. Since the drivers do not expose any system calls for use by user processes, such interaction is realised concretely by enabling the OpenSSL cryptodev extension: each encryption operation invoked is processed, by OpenSSL, via the associated cryptodev kernel module and ultimately performed by the co-processor.

Black-box architectural analysis. Treating the co-processor as a black-box, we use the functionality offered (i.e., ECB, CBC and CTR modes, with 128-, 192and 256-bit key sizes) and the extensive literature on similar hardware designs to infer the (probable) internal design. Specifically, the registers exposed (e.g., for the IV) and requirement to reinitialise the key register per invocation (so the key schedule is likely recomputed for each encryption) suggest an iterative design: as illustrated by Figure 7, a single (combinatorial) core is likely used by surrounding control logic in multiple steps to realise each mode. The default driver behaviour capitalises on hardware DMA support, via the scatter-gather mechanism, to operate autonomously from the ARM core.

Signal hunting. During any exploration phase, it is important to first establish a) an identifiable form and b) a base alignment point for the target signal; doing so maximises the chances of successfully detecting leakage. The two challenges are intrinsically linked, since a well defined form will facilitate alignment. However, the former challenge is perceived as being simple, because the target operation will typically yield a pronounced, identifiable form by virtue of how it is computed and by what. This was true, for instance, in Section 3 we were able to easily detect leakage from the ARM core by monitoring the frequency response during execution. In contrast, this is not true for the co-processor: not only is it 
unclear how AES is computed, we could not identify any periodic leakage signature linked to the AES operation. This is complicated further by virtue of the fact that the co-processor operates (semi-)independently of the ARM core (thus any hardware trigger used will be asynchronous to encryption operations).

Without any visual cues nor a reliable trigger, we were unsuccessful in detecting leakage under fixed-versus-random tests at the probe locations identified in Section 3 . Further attempts to manually scan the AM335x surface at alternative probe locations did not yield better results. However, we did manage to detect the DMA strobes by locating a probe over the memory access region: these are, of course, inherently related to encryption operations and hence (to some degree) yield a (somewhat) synchronous trigger for activity by the co-processor. The difficulty with capitalising on this fact is that any memory intensive instruction sequence can cause false positives, rendering the trigger less reliable.

To combat this issue, we instead rely on saturating the DMA engine with other work: this forces the driver into a non-DMA fall-back mode, which issues interrupts for any memory management. These interrupts are used as a trigger for AES operations on the co-processor. While less ideal than the free-running scenario in Section 3, we argue this is incrementally better than a GPIO-based hardware trigger. Specifically, it requires an attacker controlled process be coresident on the target device (cf. "spy process" in access-driven cache attacks such as [36]) rather than invasive alteration of the target process.

Testing strategy. With the trigger mechanism active, we placed several probes at various locations on the AM335x surface and repeated the same fixed-versusrandom tests as above (using all available channels on the oscilloscope, sampling at a rate of $2.5 \mathrm{GS} / \mathrm{s}$ ). Their repeated failure led us to abandon generic fixedversus-random tests, and instead focus on more specifically tailored leakage detection test vectors. A test plan was developed to target several leakage models, using semi-fixed-versus-random [6, Section 5] test vectors. The only strategy to yield detectable leakage (which we focus on subsequently as a result) was Hamming distance; the associated test vectors force a small Hamming distance between round input and output. Figure 8 shows an averaged trace and the resulting t-test result (over 10,000 traces); note that the t-statistic far exceeds our significance threshold of $\tau=4.5$.

Signal processing and detrending. Although the leakage detection step was successful, it gave us little insight into the signal characteristics. As a first step, we carried out an automated scan of the AM335x surface, performing semi-fixedversus-random tests (over 400 traces) at increments of $1 \mathrm{~mm}$ in each dimension; the location yielding the highest t-statistic will likely maximise our chances of success during key recovery. The results are visualised as a heat map in Figure 9 .

Having optimised the probe location, we applied wavelet analysis 15. (see Appendix B in an attempt to increase the SNR. There are already results [10,16] demonstrating wavelet transforms effective for filtering (denoising) and decomposition analysis in the context of side-channel attack. In general, denoising involves applying a soft-threshold [17/18] on the details components at each filter 


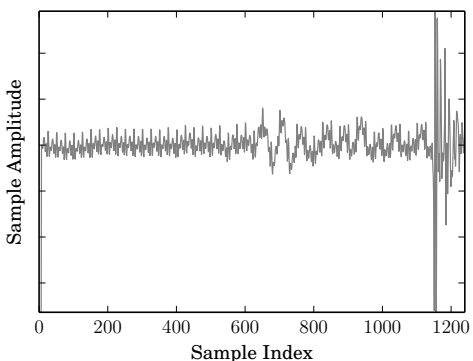

(a) Averaged trace.

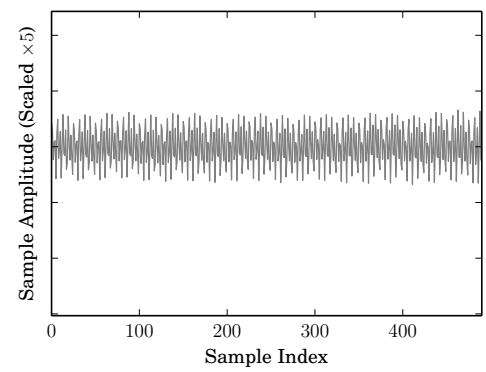

(c) Filtered trace.

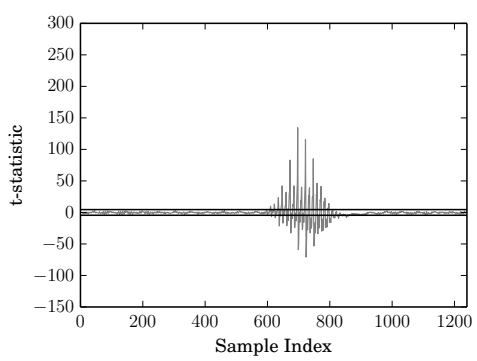

(b) Averaged t-test.

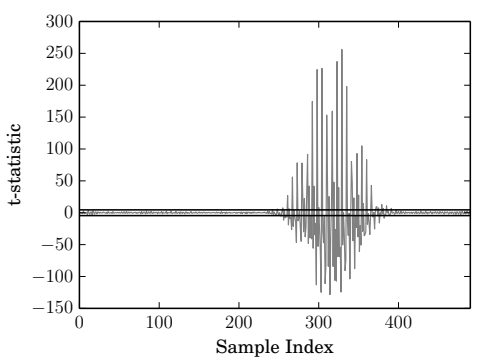

(d) Filtered t-test.

Fig. 8: Leakage detection test results for the AES co-processor running with a saturated DMA. The two columns relate to an averaged trace (left) and the related t-test result (right). The rows are indicative of raw unprocessed traces (top) and traces post-processed via the wavelet analysis (bottom).

level before resynthesising the (clean) signal. However, a high-magnitude, semicorrelated interference signal overlaid the low-magnitude signal of interest. Both signals separately had low-noise but the interference reduced the SNR of the signal of interest. As the interference contained overlapping frequencies with the signal of interest, a wavelet based detrending scheme, as used in [39], provided an effective and efficient approach for separation. After trying various wavelets, ones with a lower number of vanishing moments provided better results, indicating the need for a fast response to sudden changes in the interference [15141. The detrending technique follows a simple algorithm, inspired by the wavelet shrinkage techniques as described in [1016]. First, perform the DWT [30] with the Haar wavelet. The low ratio of the sampling rate over frequency content of the signal required only a single level computed. Then, set all of the resulting approximation coefficients to zero before performing an inverse DWT on the detail coefficients. The resulting signal (shown in Figure 8) with the interference extracted, yields a stronger result from the leakage detection test.

\subsection{Analysis and discussion}

Summarising the section above, to mount a concrete attack we performed an acquisition phase as follows: 


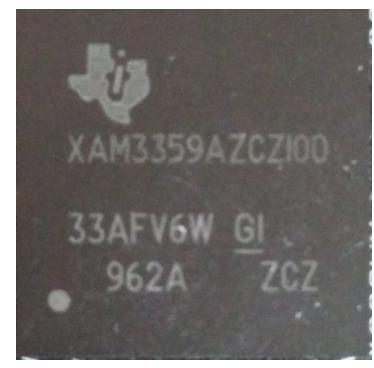

(a) AM335x surface.

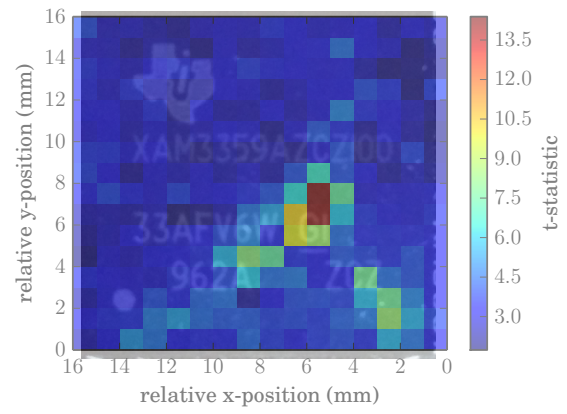

(b) AM335x surface overlaid with heat-map.

Fig. 9: An illustration of results from the automatic AM335x surface scan, using a semi-fixed-versus-random strategy to identify leakage from the cryptographic co-processor running AES-256-CBC: the t-statistic highlights the region of interest (modulo fidelity of the probe versus the dimensions in question) used as a subsequent probe location.

1. Saturate the DMA mechanism such that the driver operates in the non-DMA fall-back mode.

2. Acquire $n=500,000$ traces, each associated with 1 encryption operation and averaged over $l=1,000$ trials; match these traces with the associated ciphertexts.

3. Apply wavelet post-processing to each trace to maximise SNR.

Note that our interrupt-based trigger offers the best alignment achievable; postprocessing to improve alignment was impossible, due to the lack of an identifiable form for AES operations. The acquisition process took around 3 days. To exploit the leakage, we then applied a single-bit correlation-based attack: if $s_{i}$ denotes the AES state after $i$ iterations through the AES core, we target $\mathcal{D}\left(s_{i-1}, s_{i}\right)$ for $i=10$ relating to the final AES round. This succeeded in recovering $k$ (albeit with a modest amount of key enumeration to cope with one lower-ranked byte).

There are (at least) three important conclusions to draw from the above. First, the effort required to identify leakage from the device far outweighs that of subsequent acquisition and attack phases: only by using a rigorous leakage detection methodology were we able to get a satisfactory outcome. Second, a gap exists: although Figure 8 indicates that strong leakage is identified, our attack is unable to capitalise on this efficiently. This suggests that while leakage detection is a necessary first step, translating it into an accurate leakage model (in our case, Hamming distance seems not to be so) is also important in concrete attack scenarios. Third, while black-box analysis gave some insight into the coprocessor architecture, this did not extend to the internal implementation. In particular, the initial failure of our testing strategy suggests either a) the trigger mechanism is not accurate enough to align traces correctly, hence decimating the SNR, and/or b) the co-processor is, in some way, protected against sidechannel attacks. If the latter is true, it remains unclear which countermeasure is implemented: in contrast with Heinz et al. 24], for example, there is no structure 
in the signal that suggests time-based hiding, but equally attempted higherorder attacks on possible masking strategies were unsuccessful. Either way, if a countermeasure is implemented, then we conclude it only seems effective in increasing attack cost rather than preventing an attack.

\section{NEON}

NEON is a general-purpose SIMD extension to Cortex A-series ARM cores, harnessed, for example, by Bernstein and Schwabe 8 to both accelerate cryptographic workloads and deliver constant execution time. In terms of the ISA, each vector instruction $\odot^{w}$ processes vector operands with $l=\frac{n}{w}$ elements (or sub-words), each $w$-bits in size; $n \in\{64,128\}$ is determined by the instruction type (more specifically, whether the operands are double- or quad-words). For the simplest case of a pure vector operation, we can therefore say

$$
\boldsymbol{r}=\boldsymbol{x} \odot^{w} \boldsymbol{y} \mapsto\left\langle r^{w}[0]=x^{w}[0] \odot^{w} y^{w}[0], \ldots, r^{w}[l-1]=x^{w}[l-1] \odot^{w} y^{w}[l-1]\right\rangle
$$

where $t^{w}[j]=\boldsymbol{t}[j \cdot w, \ldots,(j+1) \cdot w-1]$ is the $j$-th $w$-bit (scalar) sub-word of vector $\boldsymbol{t}$, and $\odot^{w}$ is the operation $\odot$ for such sub-words. The ISA naturally captures standard logical (e.g., $\odot=\oplus$ with $w=1$ ) and arithmetic (e.g., $\odot=$ + with $w=8, w=16$ or $w=32$ ) operations, plus various more specialist extensions. Note that although the semantics of quad-word NEON instructions suggest they process 128-bit operands, the pipeline will in fact issue two 64-bit micro-operations.

\subsection{Instruction-level characterisation}

In this section we study leakage from (a subset of) NEON instructions by focusing on observation of

$$
\boldsymbol{r}_{i}=\mathrm{DUT}^{\odot^{w}}\left(\boldsymbol{x}_{i}, \boldsymbol{y}_{i}\right) \rightsquigarrow \lambda_{i}
$$

for a range of $\odot^{w}$ but, without loss of generality, on $w \in\{8,32\}$ bit sub-words within double-word, i.e., $n=64$ bit, operands. Given that the NEON pipeline is tightly coupled to the ARM pipeline, we reason the two will be physically close on the AM335x surface; as such, we retain the same experimental configuration (e.g., same probe location) as Section 3 However, our specific remit means we compromise by using a strictly controlled profiling device: a hardware trigger is used throughout to support instruction-level (i.e., cycle-accurate) alignment and hence a lower bound on success rate.

Leakage detection. We performed an initial exploration focused on a limited, indicative set of NEON instructions: the aim was to gather general intuition about their leakage characteristics. As such, we considered various potential sources of leakage. Consider, for example, execution of a vector XOR instruction (e.g., veor. u32 d0, d1, d2): one could potentially observe leakage related to operand reads (i.e., from d1 and/or d2), computation of the operation, or result write-back (i.e., to do). 

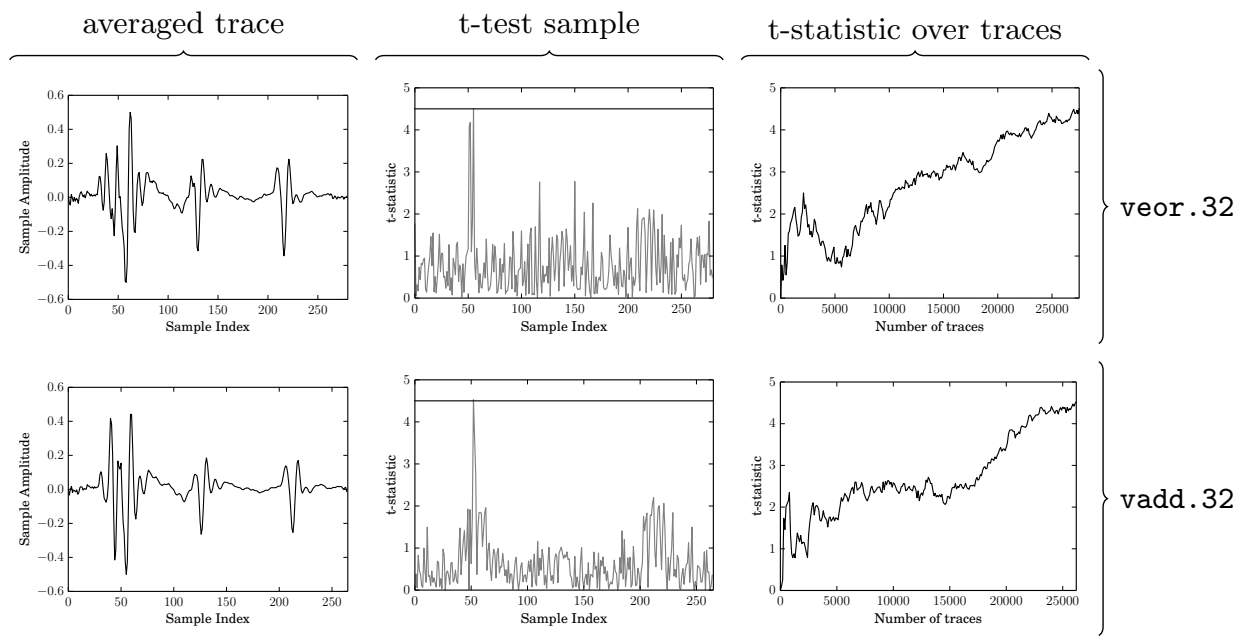

vadd. 32
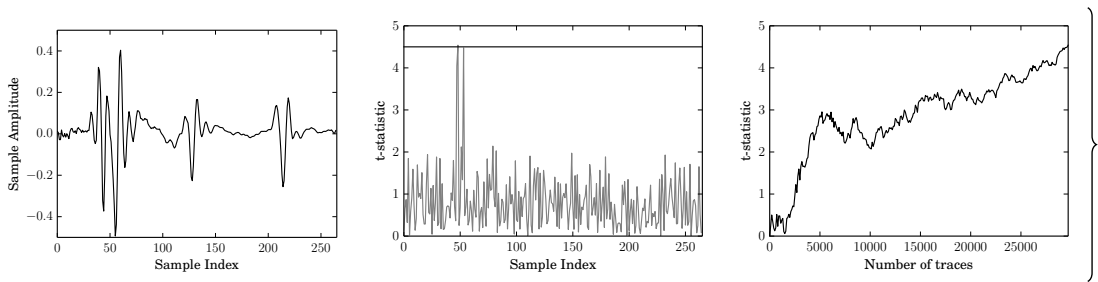

vmul. 32
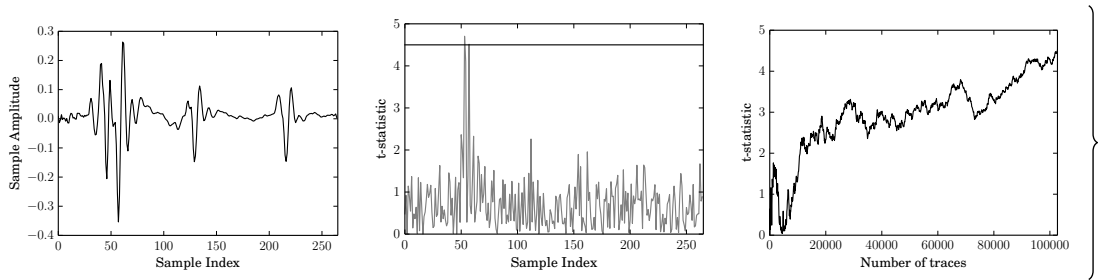

vceq. 32

Fig. 10: t-statistic over number of traces for fixed-versus-random tests on NEON instructions. In top-down order: veor.u32, vadd.u32 and vmul.u32, vceq.u32. 
In summary, the results (as illustrated in Figure 10 show that a) clear operation-dependent SPA leakage is evident, allowing, for example, construction of per-instruction templates, and b) data-dependent leakage is evident, but from result write-back only: we could identify no leakage relating to operand reads. The latter fact, i.e., the statistically observable difference between writeback of random versus fixed results, confirms that the leakage point relates to said step (not reading operands from memory).

Comparing the ARM and NEON cores. Section 3 already shows that exploitable leakage from the ARM core exists; our next goal was to roughly quantify the difference between it and the NEON core. In summary, applying the same leakage detection strategy (as illustrated in Figure 11) shows that on the ARM core a) leakage from the operation plus both operand reads and result write-back is evident, and b) the strength of that leakage (in terms of the t-statistic growth per sample count) is greater than on the NEON core (especially for the integer multiplication instruction). Explaining this difference seems difficult without detailed knowledge of the implementation(s). However, it suggests a difference in the arithmetic (e.g., multiplier) design used.

Hamming weight leakage. Having identified a set of leakage points, our next goal was to analyse and exploit their structure. More specifically, we attempted to align the characterisation with standard attacks by tracking the Hamming weight of results written-back against associated leakage. This is achieved by amending the fixed-versus-random methodology, so semi-fixed test vectors are selected (for a given Hamming weight).

The results of this analysis, plus their utility, are discussed in the following sections. We stress that, throughout, the Hamming weight of the entire $n$-bit result is considered: our results show that focusing on an individual $w$-bit subword is feasible, but with the expected increase the number of traces. That is, if one considers leakage with regards to a single $w$-bit sub-word then the other $l-1$ sub-words can be considered noise (thus overcome by acquiring more traces).

Arithmetic and logical operations. Figure 12 illustrates leakage from two specific NEON instructions pertinent to cryptography; further examples are shown in Figure 13 The (vector) XOR case is indicative of most instructions, in the sense that clear separation between distinct Hamming weights is evident. In contrast, the vector polynomial multiplication is something of a special case. The separation between distinct Hamming weights is still evident, and potentially relevant to cryptographic use-cases (e.g., [9]). However, unlike the other instructions there is some "cross over" with regard to the Hamming weight and signal that we cannot currently explain.

Comparison operations. Figure 14 illustrates leakage from a NEON vector comparison instruction. In contrast to a scalar comparison on the ARM core (which produces a 1-bit result in the CPSR status register), a NEON vector comparison sets (or clears) all $w$ bits in each sub-word to signal true or false (i.e., forms a 

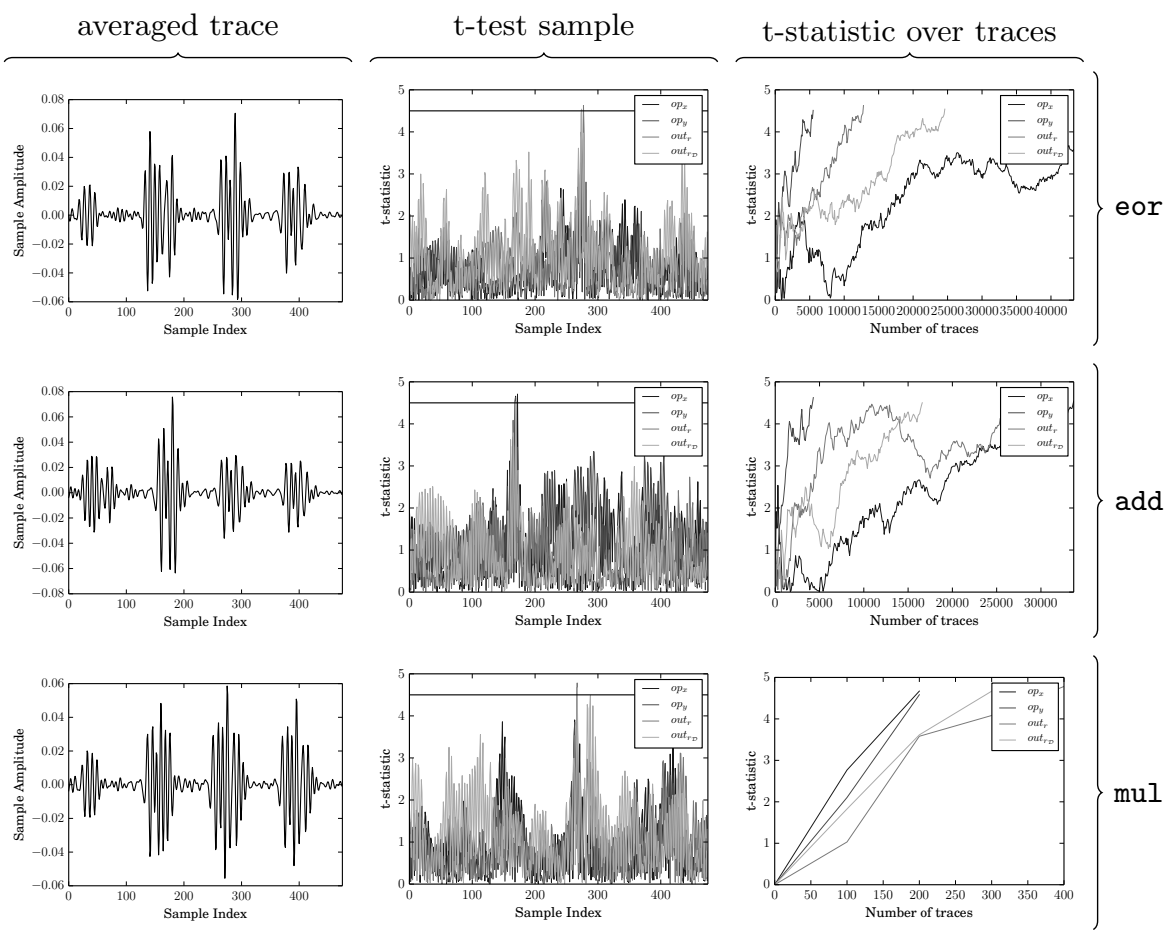

Fig. 11: t-statistic over number of traces for fixed-versus-random tests on ARM instructions. In top-down order: eor, add and mul. 


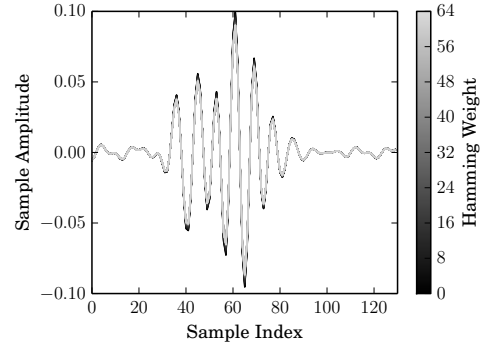

(a) veor.u32 instruction (raw).

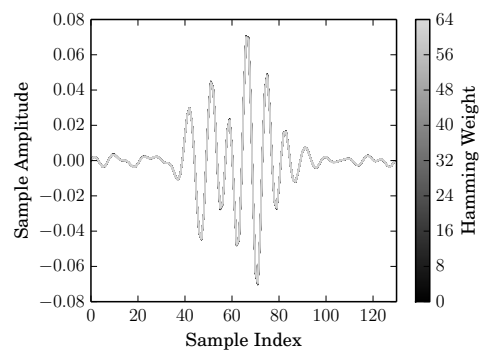

(c) vmul.p8 instruction (raw).

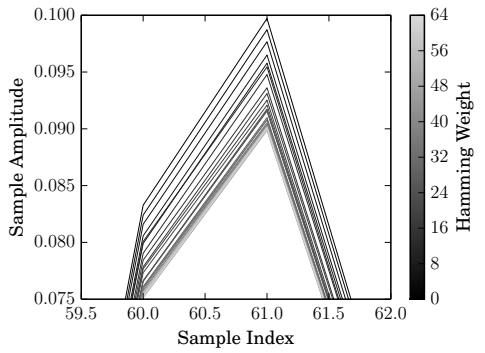

(b) veor.u32 instruction (zoom).

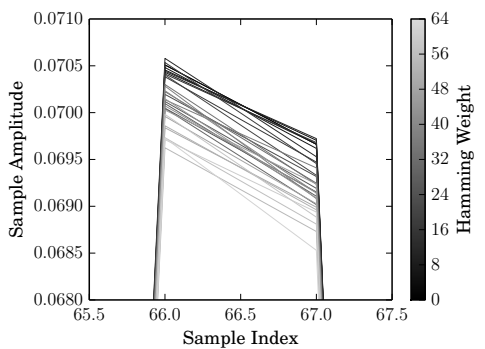

(d) vmul.p8 instruction (zoom).

Fig. 12: Illustration of Hamming weight leakage for a (limited) set of NEON arithmetic and logical instructions (where $w=32$ ).

mask). Without loss of generality, we focus on equality comparison:

$$
r^{w}[j]=\left\{\begin{aligned}
2^{w}-1 & \text { if } x^{w}[j]=y^{w}[j] \\
0 & \text { otherwise. }
\end{aligned}\right.
$$

This can be used to support branch-free, constant-time implementations: the resulting mask is used to control conditional execution of subsequent operations in each sub-word, replacing conditional control-flow by conditional data-flow.

Our results demonstrate two important facts. First, analysis of leakage from a vector comparison reveals the (total) number of sub-word results that were true (or false); this is as expected, given the maximal and minimal Hamming weight of the outputs (i.e., masks) produced in each case. Second, as demonstrated by Figure 16, it is possible to target a specific sub-word, and hence ascertain whether it has the value $2^{w}-1$ or 0 . Doing so means considering each sub-word independently, treating the remaining sub-words as noise (cf. single-bit DPA).

\subsection{A concrete attack on AES}

The charactisation above clearly suggests Hamming weight leakage can be leveraged in concrete attacks. As justification, we consider a scenario where the attacker can observe computation of

$$
m_{i}=\mathrm{DUT}_{k}^{\mathrm{AES}-128-\mathrm{CBC}}\left(c_{i}\right) \rightsquigarrow \lambda_{i}
$$



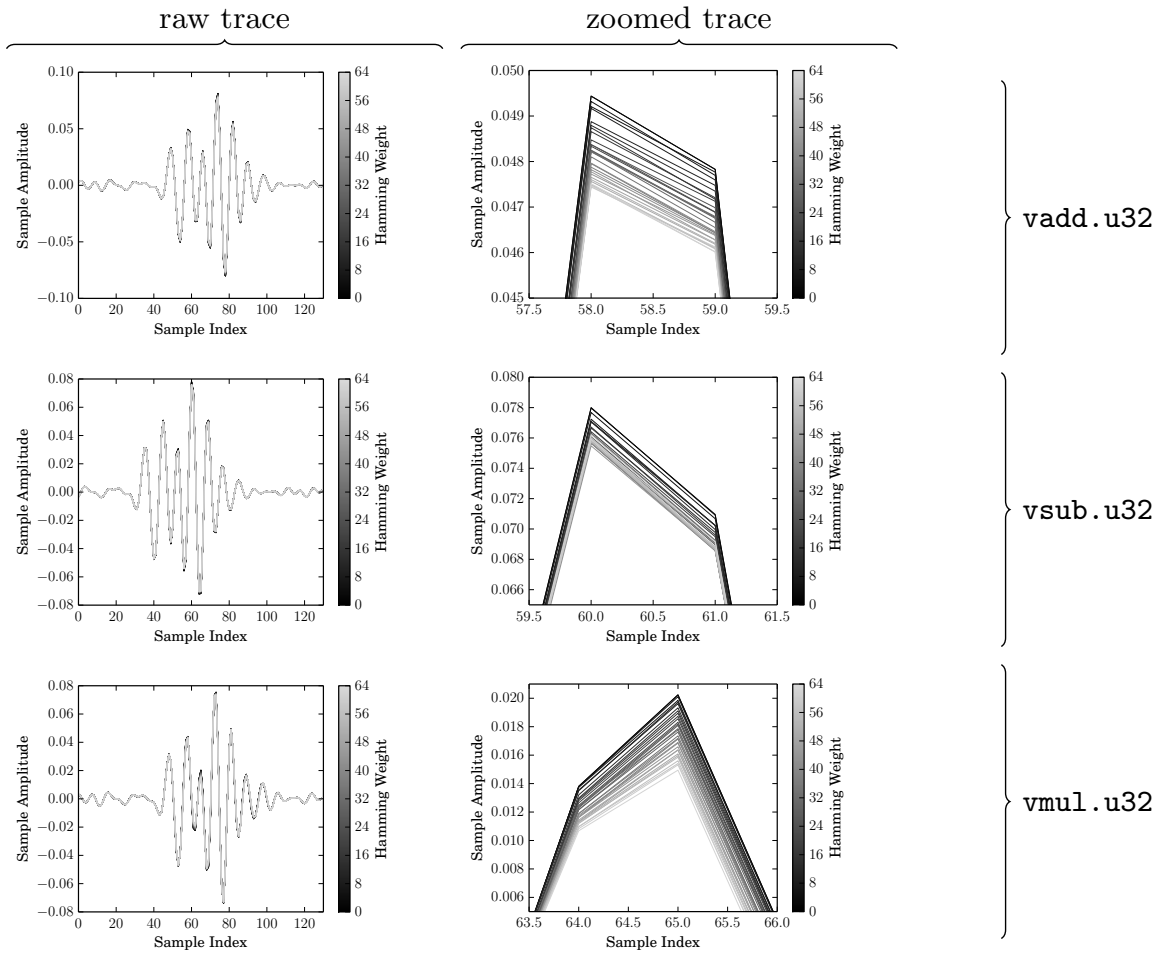

Fig. 13: Illustration of Hamming weight leakage for a (limited) set of NEON arithmetic and logical instructions (where $w=32$ ). 


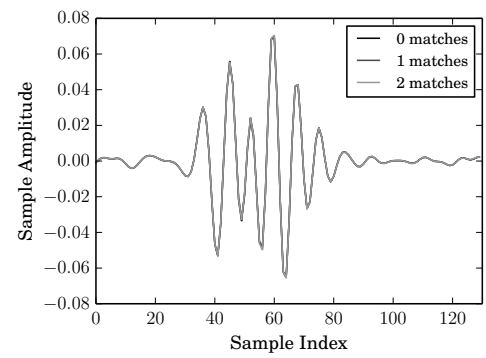

(a) vceq.u32 instruction (raw).

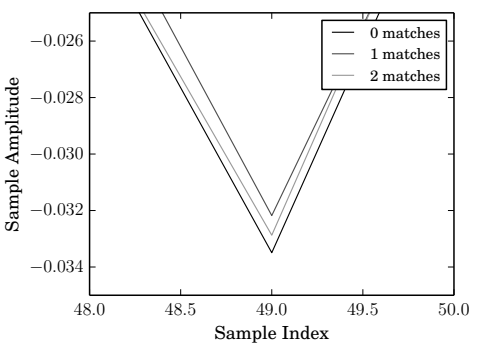

(b) vceq.u32 instruction (zoom).

Fig. 14: Illustration of Hamming weight leakage for NEON comparison instructions (where $w=32$ ).

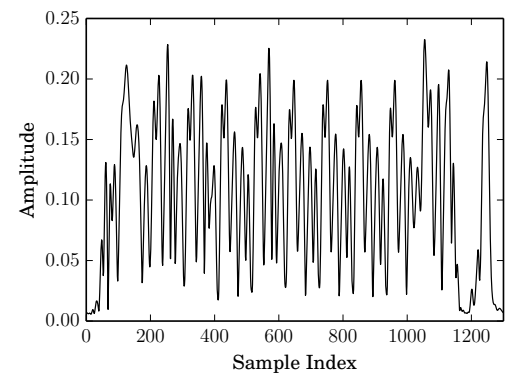

(a) Single averaged trace.

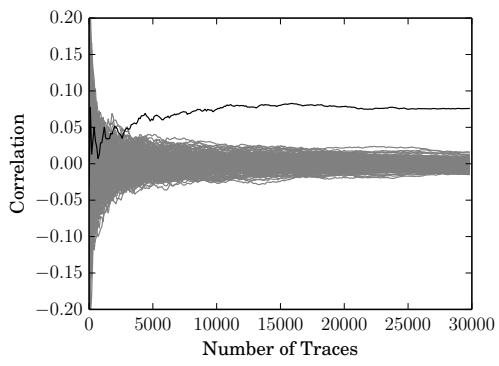

(b) Single byte correlation.

Fig. 15: Illustration of decryption (for a 128-byte ciphertext) using the NEONbased bit-sliced implementation of AES in OpenSSL; the corresponding correlation coefficient evolution suggests the attack succeeds with $\sim 5,000$ traces.

but alter how AES itself is realised (compared with Section 3 and Section 4): we instead target the NEON-based bit-sliced implementation in OpenSSL (which stems from work by Käsper and Schwabe [27, and was enabled via the preprocessor flag -DBSAES_ASM).

This particular implementation is triggered if $c_{i}$ is sufficiently large (namely 128 bytes, falling-back to an alternative implementation otherwise). Although we note the techniques in Section 3 remain broadly applicable, for clarity we retain the same experimental environment as Section 5.1 (e.g., with a hardware trigger). The attack then proceeds in a fairly straightforward manner: we simply use key (byte, due to the representation of data used by the implementation) hypotheses based on Hamming weight of the intermediate state after the first round InvSubBytes operation in a standard, correlation-based approach. The attack succeeds with $\sim 5,000$ traces, requiring $5000 \cdot 128=625 \mathrm{kB}$ of ciphertext.

\subsection{A theoretical attack on NORX}

Perhaps more so than other operations, the relevance of leakage from a vector comparison needs motivation. As such, consider NORX [4], an AEAD-based 


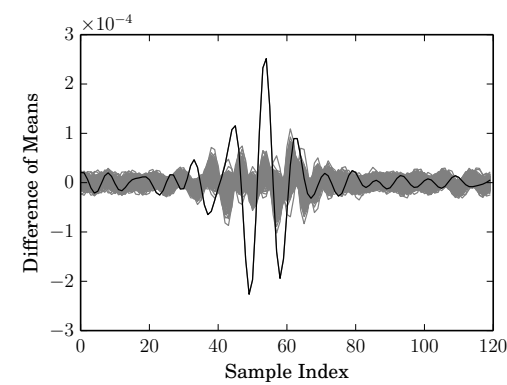

(a) Sample difference.

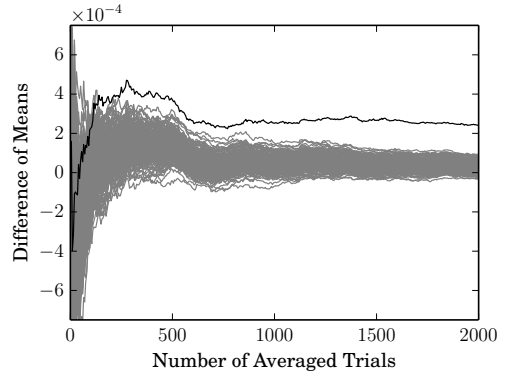

(b) Difference over averaged trials.

Fig. 16: Illustration of a single-word attack on vceq.u32: the black hypothesis for $r^{w}[j]=2^{w}-1$ (i.e., where the comparison is true, without loss of generality for $j=0$ ) is clearly distinguished after averaging over 1,000 trials.

CAESAR candidate whose reference implementation 8 harnesses NEON. The NORX32-6-1 parametrisation (i.e., for 32-bit word size, 6 rounds, parallelism degree 1 , and 128-bit tag size) verifies tags as follows

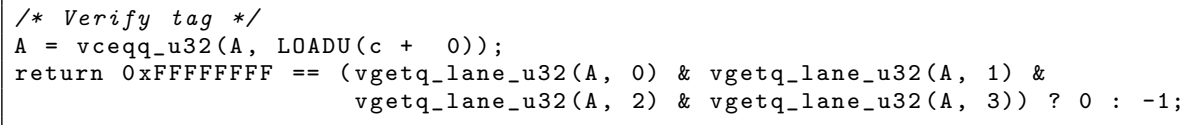

noting the state A (representing the computed tag) is compared with the received tag using vector comparison on 32 -bit sub-words.

On one hand this is attractive since a) it is likely more efficient than four sequential 32-bit comparisons, and b) it is constant-time, unlike an alternative such as use of memcmp. On the other hand, consider a (purely hypothetical) scenario where an attacker has access to a decryption oracle, i.e., execution of

$$
\mathrm{DUT}_{k}^{\mathrm{NORX} 32-6-1}\left(c_{i}\right) \rightsquigarrow \lambda_{i}
$$

for chosen $c_{i}$ can be observed. The resulting leakage can be used to enable tag forgery: the attacker is able to determine the total number of matching sub-words for a candidate tag, so requires $O\left(2^{32}\right)$ queries (albeit with a constant factor that hides the cost of dealing with noise etc. in acquisitions) to brute-force search for a tag matching ciphertext of their choice. Whether or not such an approach is feasible in practice clearly depends on the context, but equally clear is the gap between this and the supposed (theoretical) security level.

\section{Conclusions}

Summary of results. In this paper we present concrete, EM-based analysis of the AM335x SoC and software executing on it. Although hard to compare directly, a

${ }^{8}$ See http://github.com/norx/NORX We stress our analysis should in no way be inferred as criticism of NORX within the context of CAESAR. 


\begin{tabular}{|c|c|c|c|c|c|c|}
\hline Section & Operation & Implementation & Hardware & Trigger & Acquisitions & Data \\
\hline 3 & Decryption & T-tables & ARM core & GPIO-based & 3,000 & $46 \mathrm{kB}$ \\
\hline 3 & \begin{tabular}{|l|} 
Encryption \\
\end{tabular} & T-tables & ARM core & Network-based & 100 & $400 \mathrm{kB}$ \\
\hline 4 & Encryption & Hardware & Co-processor & DMA-based & 500,000 & $7 \mathrm{~GB}$ \\
\hline 5 & Decryption & Bit-sliced & NEON core & GPIO-based & 5,000 & $625 \mathrm{kB}$ \\
\hline
\end{tabular}

Table 1: Summary of results.

summary of our results targetting CBC-based AES is shown in Table 1. Beyond this, however, we draw several more general conclusions:

1. Despite suggestions to the contrary [32], higher clock frequency does not imply a requirement for a high sampling rate: instruction- or cycle-level resolution may yield better results, but we still observed exploitable leakage at much lower frequencies (mirroring observations such as in [22]). This fact refutes any suggestion that the impact of EM-based attacks (against targets of this complexity) is lessened by cost: for example, in Section 3 a (postcharacterisation, i.e., excluding the XY-table) attack can be mounted using $\sim \$ 1,800$ of equipment (including a suitable low-end oscilloscope in place of the digitiser, and hand-made 30 AWG coil, 15-turn probe).

2. For devices of this complexity, the value offered by the TVLA methodology is significant: we suggest that an ad hoc analysis would have been difficult or ineffectual, particularly in the context of an unknown target (as in Section 4).

3. Target complexity suggested at a high level does not imply the same complexity at lower levels. Our results demonstrate this fact in two examples. First, we were able to bypass complexity relating to the SoC architecture or fabrication technology by targeting support circuitry in Section 3 . Second, in Section 5, we observed that parallelism suggested in the NEON ISA (traditionally viewed as a complicating factor in DPA, for example) is not realised in the micro-architecture: This is of relevance to constructions (e.g., 33, albeit studied in the context of hardware) that rely on parallelism somehow.

4. Although the drive for efficient, constant-time implementation using NEON is clearly important, it seems prudent to proactively consider when/how such an approach can enable other forms of leakage. For example, Section 5 demonstrates that vector comparison, while advantageous in the sense of having a fixed latency, will still leak information about sub-word (in)equality.

5. Software such as OpenSSL is now becoming commonplace in (embedded) scenarios in which it might traditionally have been deemed too heavy-weight. As a result, alongside the challenging goal of securing such software against network-based attacks, it is starting to seem of long-term importance that countermeasures for hardware side-channels are also proactively considered.

Future work. Our limited scope suggests various items of future work; beyond obvious directions, such as analysis of asymmetric cryptography on the platform, two stand out. First, a rigorous analysis of all constituent components is of general value. For example, although we deemed the ${ }^{9} \mathrm{SHA}, \mathrm{RNG}$ and support out of

\footnotetext{
${ }^{9}$ PKA is assumed to stand for Public-Key Accelerator.
} 
scope, understanding their leakage characteristics seems important. In the same way, an exhaustive analysis of the NEON unit was impractical in this paper (due to space), even though the techniques easily extend to other instructions. Doing so, and, in particular, understanding vector instructions with no natural scalar equivalent, also seem important. Second, improvements in analysis techniques could incrementally improve specific results. For example, in Section 3 the number of acquisitions required to perform a successful key recovery attack is partly dictated by how effective the pre-processing is: we expect some effort to narrow the gap between results for the artificial and free-running cases. Likewise, Section 4 highlights the challenge of translating a method of leakage detection into a leakage model; resolving this issue in the black-box setting seems difficult, but clearly important in scenarios such as the one investigated.

\section{Acknowledgements}

Jake Longo has been supported in part by a studentship under the EPSRC Doctoral Training Partnership (DTP) scheme. The authors would like to thank Pankaj Rohatgi for general discussion, and Sami Saab for specific help with signal processing/analysis. We also thank both Billy Brumley and Markku Saarinen for their insight on NEON-based implementation, Martijn Stam for discussion about AEAD, and the NORX team, all of who help improved Section 5 .

\section{References}

1. D. Aboulkassimi, M. Agoyan, L. Freund, J.J.A. Fournier, B. Robisson, and A. Tria. ElectroMagnetic Analysis (EMA) of software AES on Java mobile phones. In Information Forensics and Security (WIFS), pages 1-6, 2011.

2. D. Aboulkassimi, J.J.A. Fournier, L. Freund, B. Robisson, and A. Tria. EMA as a physical method for extracting secret data from mobile phones. IJCSA, 2(1):16-25, 2013.

3. D. Agrawal, B. Archambeault, J.R. Rao, and P. Rohatgi. The EM side-channel(s). In CHES, pages 29-45. LNCS 2523, 2003.

4. J.-P. Aumasson, P. Jovanovic, and S. Neves. NORX. CAESAR submission specification, version 1.1, 2014. http://norx.io/data/norx.pdf

5. J. Balasch, B. Gierlichs, R. Verdult, L. Batina, and I. Verbauwhede. Power analysis of Atmel CryptoMemory - recovering secret keys from secure EEPROMS. In $C T$ $R S A$, pages 19-34. LNCS 7178, 2012.

6. G.T. Becker, J. Cooper, E. DeMulder, G. Goodwill, J. Jaffe, G. Kenworthy, T. Kouzminov, A. Leiserson, M. Marson, P. Rohatgi, and S. Saab. Test Vector Leakage Assessment (TVLA) methodology in practice. In ICMC, 2013.

7. G.T. Becker, M. Kasper, A. Moradi, and C. Paar. Side-channel based watermarks for IP protection. In COSADE, pages 47-50, 2010.

8. D.J. Bernstein and P. Schwabe. NEON crypto. In CHES, pages 320-339. LNCS 7428, 2012.

9. D. Câmara, C.P.L. Gouvêa, J. López, and R. Dahab. Fast software polynomial multiplication on ARM processors using the NEON engine. In $C D-A R E S$, pages 137-154. LNCS 8128, 2013. 
10. X. Charvet and H. Pelletier. Improving the DPA attack using wavelet transform. In NIST Physical Security Testing Workshop, 2005.

11. T. Chothia and A. Guha. A statistical test for information leaks using continuous mutual information. In CSF, pages 177-190, 2011.

12. O. Choudary and M.G. Kuhn. Efficient template attacks. In CARDIS, pages 253-270, 2013.

13. O. Choudary and M.G. Kuhn. Template attacks on different devices. In COSADE, pages 179-198, 2014.

14. J. Daemen and V. Rijmen. The Design of Rijndael. Springer, 2002.

15. I. Daubechies. Ten Lectures on Wavelets. CBMS-NSF Regional Conference Series in Applied Mathematics. Society for Industrial and Applied Mathematics, 1992.

16. N. Debande, Y. Souissi, M.A.E. Aabid, S. Guilley, and J. Danger. Wavelet transform based pre-processing for side channel analysis. In MICROW, pages 32-38, 2012.

17. D.L. Donoho. De-noising by soft-thresholding. IEEE Transactions on Information Theory, 41(3):613-627, 1995.

18. D.L. Donoho and I.M. Johnstone. Ideal spatial adaptation by wavelet shrinkage. Biometrika, 81(3):425-455, 1994.

19. D. Du, S. Narasimhan, R. Subhra Chakraborty, and S. Bhunia. Self-referencing: A scalable side-channel approach for hardware Trojan detection. In CHES, pages 173-187. LNCS 6225, 2010.

20. T. Eisenbarth, T. Kasper, A. Moradi, C. Paar, M. Salmasizadeh, and M.T. Manzuri Shalmani. On the power of power analysis in the real world: A complete break of the KeeLoq code hopping scheme. In CRYPTO, pages 203-220. LNCS 5157, 2008.

21. K. Gandolfi, C. Mourtel, and F. Olivier. Electromagnetic analysis: Concrete results. In CHES, pages 251-261. LNCS 2162, 2001.

22. D. Genkin, L. Pachmanov, I. Pipman, and E. Tromer. Stealing keys from PCs by radio: Cheap electromagnetic attacks on windowed exponentiation. Cryptology ePrint Archive, Report 2015/170, 2015. http://eprint.iacr.org/

23. G. Goodwill, B. Jun, J. Jaffe, and P. Rohatgi. A testing methodology for sidechannel resistance validation. In NIST Non-Invasive Attack Testing Workshop, 2011.

24. B. Heinz, J. Heyszl, and F. Stumpf. Side-channel analysis of a high-throughput AES peripheral with countermeasures. In ISIC, pages 25-29, 2014.

25. Cryptography Research Inc. Test Vector Leakage Assessment (TVLA) Derived Test Requirements (DTR) with AES. http://www.cryptography.com/public/pdf/TVLA-DTR-with-AES.pdf.

26. Texas Instruments. AM335x Sitara processor datasheet. Technical Report SPRS717G, TI, 2014. http://www.ti.com/lit/ds/symlink/am3358.pdf.

27. E. Käsper and P. Schwabe. Faster and timing-attack resistant AES-GCM. In CHES, pages 1-17. LNCS 5747, 2009.

28. M. Kasper, T. Kasper, A. Moradi, and C. Paar. Breaking KeeLoq in a flash: On extracting keys at lightning speed. In AFRICACRYPT, pages 403-420. LNCS 5580, 2009.

29. P.C. Kocher, J. Jaffe, and B. Jun. Differential power analysis. In CRYPTO, pages 388-397. LNCS 1666, 1999.

30. S.G. Mallat. A theory for multiresolution signal decomposition : the wavelet representation. IEEE Transactions on Pattern Analysis and Machine Intelligence, 11(7):674-693, 1989.

31. S. Mangard, E. Oswald, and T. Popp. Power analysis attacks: Revealing the secrets of smart cards. Springer, 2008. 
32. E. Mateos and C.H. Gebotys. Side channel analysis using Giant Magneto-Resistive (GMR) sensors. In COSADE, pages 42-49, 2011.

33. M. Medwed, F.-X. Standaert, and A. Joux. Towards super-exponential side-channel security with efficient leakage-resilient PRFs. In CHES, pages 193-212. LNCS 7428, 2012.

34. A. Moradi, A. Barenghi, T. Kasper, and C. Paar. On the vulnerability of FPGA bitstream encryption against power analysis attacks: extracting keys from Xilinx Virtex-II FPGAs. In CCS, pages 111-124, 2011.

35. A. Pellegrini, V. Bertacco, and T. Austin. Fault-based attack of RSA authentication. In DATE, pages 855-860, 2010.

36. C. Percival. Cache missing for fun and profit, 2005. http://www.daemonology. net/papers/htt.pdf

37. J.-J. Quisquater and D. Samyde. ElectroMagnetic Analysis (EMA): Measures and counter-measures for smart cards. In E-SMART, pages 200-210. LNCS 2140, 2001.

38. P. Rohatgi. Electromagnetic attacks and countermeasures. In Cryptographic Engineering, pages 407-430. Springer, 2009.

39. S. Saab, A. Leiserson, and M. Tunstall. Efficient key extraction from the primary side of a switched-mode power supply. Cryptology ePrint Archive, Report 2015/512, 2015. http://eprint.iacr.org/

40. J. Scahill and J. Begley. iSpy: The CIA campaign to steal Apple's secrets. The Intercept, 2015. http://firstlook.org/theintercept/2015/03/10/ ispy-cia-campaign-steal-apples-secrets/

41. G. Strang and G.J. Fix. An Analysis of the Finite Element Method. Automatic Computation. Prentice-Hall, 1973.

42. H. Uno, S. Endo, Y. Hayashi, N. Homma, and T. Aoki. Chosen-message electromagnetic analysis against cryptographic software on embedded OS. In $E M C$, 2014.

43. A. Zajic and M. Prvulovic. Experimental demonstration of electromagnetic information leakage from modern processor-memory systems. IEEE Transactions on Electromagnetic Compatibility, 56(4):885-893, 2014.

\section{A TVLA plan and test vector generation}

Throughout the paper, we make use of the Test Vector Leakage Assessment (TVLA) methodology [25|6|23] for leakage detection and analysis. This Appendix aims to clarify how the associated test vectors were generated, hence adding detail to discussion within the paper itself.

\section{A.1 TVLA as per Section 3}

Since OpenSSL is an open source project, we applied white-box analysis of the source code to identify potential attack vectors. By default, OpenSSL uses a T-tables-based [14, Section 4.2] implementation of AES; this is realised by an automatically generated assembly language program. Since no countermeasures are implemented against hardware (versus timing) related leakage, we did not need to apply any special-purpose leakage detection tests beyond those already described in Section 2.3. 


\begin{tabular}{|l|r|r|}
\hline Test Description & Result & $\begin{array}{r}\text { Trace } \\
\text { count }\end{array}$ \\
\hline \hline Round 7, round Hamming distance, 11 bytes @ 0x00 & Leak & 3,000 \\
\hline Round 7, round Hamming distance, 6 bytes @ 0x00 & Leak & 25,000 \\
\hline Round 7, round Hamming distance, 3 bytes @ 0x00 & No Leak & 10,000 \\
\hline Round 7, round Hamming distance, byte 1 @ 0x00 & No Leak & 10,000 \\
\hline Round 7, fixed round output, byte 1 @ 0x00 & No Leak & 10,000 \\
\hline Round 7, S-box out, byte 1 @ 0x00 & No Leak & 10,000 \\
\hline Round 7, round Hamming distance, byte 1 @ 0xFF & No Leak & 10,000 \\
\hline Round 7, round Hamming distance (before AddRoundKey, byte 1 @ 0x00 & No Leak & 10,000 \\
\hline Round 7, round Hamming distance, byte 12 @ 0x00 & No Leak & 10,000 \\
\hline Round 7, round Hamming distance, byte 4 @ 0x00 & No Leak & 10,000 \\
\hline Round 7, fixed output, byte 12 (before AddRoundKey) @ 0x00 & No Leak & 10,000 \\
\hline Round 14, S-box out, byte 1 @ 0x00 & No Leak & 10,000 \\
\hline Round 7, fixed state byte @ 0x00 & No Leak & 10,000 \\
\hline Round 7, fixed state short @ 0x00 & No Leak & 10,000 \\
\hline Round 7, fixed state word @ 0x00 & No Leak & 10,000 \\
\hline Round 7, fixed state 64-bit @ 0x00 & No Leak & 10,000 \\
\hline Three round Hamming distance @ 7-9, multiple bytes @ 0x00 & Leak & 200 \\
\hline Three round Hamming distance @ 6-8, multiple bytes @ 0x00 & Leak & 200 \\
\hline Round 7, S-box in/out Hamming distance minimised & No Leak & 1,000 \\
\hline Round 7, S-box in/out Hamming distance maximised & No Leak & 1,000 \\
\hline Round 7, S-box output 16 bytes @ 0x00 & No Leak & 1,000 \\
\hline
\end{tabular}

Table 2: TVLA test plan, and results, as discussed in Section 4 . Note that traces are averaged over 1,000 trials (i.e., 1,000 traces equates to 1 million trials).

\section{A.2 TVLA as per Section 4}

In sharp contrast with OpenSSL, the AM335x cryptographic co-processor is a proprietary design: with no explicit documentation, we were forced to apply (informed) black-box analysis and hence develop a test plan to identify potential leakage points. Table 2 lists (a subset of) the test vectors used, which were developed according to the recommendations by Becker et al. 6 and the AESspecific TVLA requirements [25]; leakage is deemed to be evident if the t-statistic associated with a given test vector exceeds $\tau=4.5$.

A set of test vectors are designed to trigger specific behaviour in AES that may lead to leakage detection. For example, the test "Round 7, round Hamming distance, 11 bytes @ 0x00" will fix (the same) 11 bytes in $\mathcal{D}\left(s_{(6)}, s_{(7)}\right)$ to zero whilst the remaining 5 bytes vary. Note, that for some vectors this may result in some intermediate round functions also being fixed. Each of the vectors are run on the hardware co-processor operating in AES-256-CBC mode.

\section{A.3 TVLA as per Section 5}

Ensuring isolation of NEON core versus ARM core and memory access. We identified leakage related to memory access early in our analysis, and assumed thereafter that any and all such operations could be compromised: this includes 


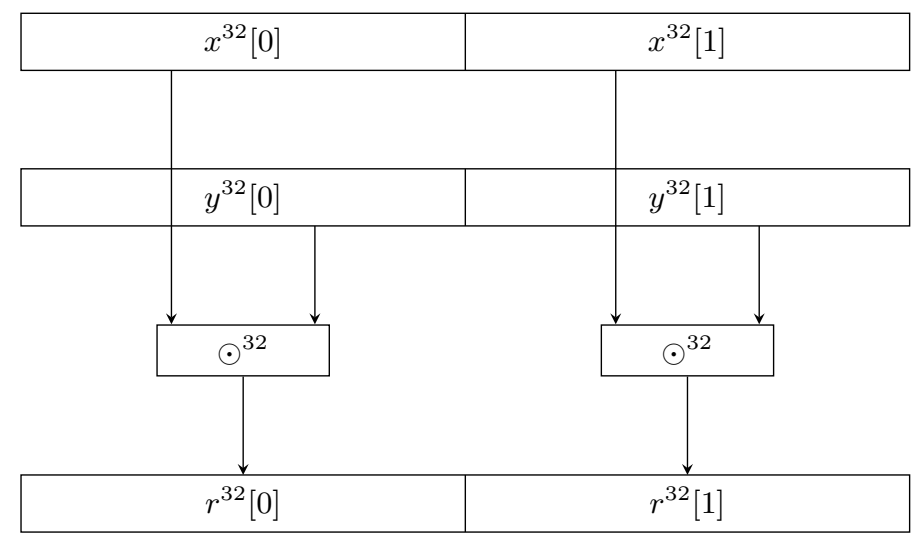

Fig. 17: An illustration of the NEON instruction $\boldsymbol{r}=\boldsymbol{x} \odot \odot^{32} \boldsymbol{y}$.

memory access by the NEON core. Likewise, our analysis showed that instructions executed by the ARM core exhibited strong leakage. A subtle implied issue is that we need to ensure that the leakage observed was solely from the instructions being studied (as executed by the NEON core, versus leakage related memory access for example). To do so, we placed an adequate NOP sled on either side of the instruction being studied. We concede that this limits the scope of our analysis to exclude cross-instruction behaviour, but feel that this is an acceptable trade-off based on our original remit.

NEON fixed-versus-random test vector generation. We target four modes of leakage for the NEON processor, namely, the input operands $\left(o p_{x}, o p_{y}\right)$, the output $\left(\right.$ out $\left._{r}\right)$ and the output write-back stage $\left(o u t_{r_{\mathcal{D}}}\right)$. Furthermore, care needs to be taken to primarily capture the leakage for a single lane of each instruction. We ran extensive analysis for all data widths $(8,16,32,64)$ where possible, however, we primarily discuss results related to the 32 -bit data-width case (see Figure 17 and Table 3). The leakage detection results showed no discernible leakage for either input operands and relatively little difference between out $t_{r}$ and out $_{r_{\mathcal{D}}}$. This suggests the leakage is primarily a function of the output bits in $r$. This is perhaps unsurprising as we often find that the leakage is closely related to the Hamming weight of a particular state.

Hamming weight analysis of NEON instructions. We focused on the Hamming weight analysis of the NEON instructions on the output of the selected instructions for 32-bit widths. We generated a set of 10,000 vectors for each Hamming weight and (in a random order) executed the vectors, whilst recording the sidechannel leakage. We applied post-processing on the traces to remove any erroneous data and sorted the traces according to their respective Hamming weights. The traces for each Hamming weight were averaged and plotted as shown in Figure 12,13 and 14 . 


\begin{tabular}{|c||c|c|c|l|}
\cline { 2 - 5 } \multicolumn{1}{c|}{} & \multicolumn{3}{c|}{ Registers } & \multirow{2}{*}{ Notes } \\
\cline { 2 - 5 } \multicolumn{1}{c||}{} & $x$ & $y$ & random & \\
\hline \hline op $_{x}$ & fixed & random & random & \\
\hline out $_{r}$ & pseudorandom & pseudorandom & fixed & $\begin{array}{l}r=x \odot^{32} y . \\
r \text { randomised } \\
\text { prior to execution. }\end{array}$ \\
\hline out $_{r_{\mathcal{D}}}$ & pseudorandom & pseudorandom & fixed & $\begin{array}{l}r=x \odot^{32} y . \\
r \text { fixed prior to } \\
\text { execution. }\end{array}$ \\
\hline
\end{tabular}

Table 3: TVLA test models for NEON operations, as discussed in Section 5 .

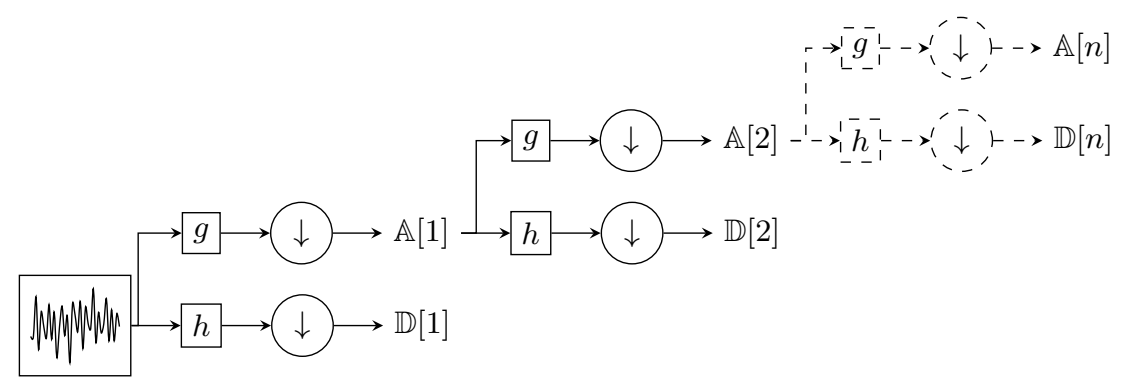

Fig. 18: An $n$-level DWT filter bank.

\section{B Wavelet analysis}

Wavelet analysis is a powerful method of analysing the time-frequency content of a signal with respect to a wavelet function (the mother wavelet $\psi$ ). Specifically, the signal is described as a function (shifting and dilation) of the mother wavelet. This offers advantages over a short-time Fourier transform, which has a limited frequency resolution based on the window size used. Generally, wavelet transforms are divided into two approaches, namely the Continuous Wavelet Transform (CWT) and the Discrete Wavelet Transform (DWT).

Continuous Wavelet Transform. The CWT is the convolution of a signal $f(t)$ with a set of transformations (shifting and dilation) of the mother wavelet. More formally, the CWT for a sampled signal $f(t)$ is defined as:

$$
C W T(d, s)=\frac{1}{\sqrt{d}} \int f(t) \psi\left(\frac{t-s}{d}\right) d t
$$

where $d$ and $s$ are the dilation and shift factors of the mother wavelet. The shift factor describes the shift in time of the mother wavelet. The dilation factor influences the mother wavelet length and hence the frequency information. In short, $d$ is inversely proportional to the frequency information carried by the transformation. We note that the CWT results in a high-resolution description about the signal $f(t)$ but at a cost of redundant transformations. 
Discrete Wavelet Transform. The DWT is similar to the Fourier transform in how it decomposes a signal into a set of terms of a basis set of functions. However, the key difference is that the Fourier transform basis functions consist of sines and cosines, whereas the DWT basis functions are a set of mutually orthogonal wavelets generated from the mother wavelet. In addition, the DWT will use two parameters in the expansion, i.e. the dilation and shift factors. In effect, the DWT acts as a filter bank and the depth of the bank describes the level of the transform.

At each level of the filter bank, the frequency content of a discrete time signal is split by way of a low and high pass filter $g$ and $h$ respectively. The output from each of the filters now contains half the frequency information previously present. Following Nyquist, we can down-sample the data and remove half the points in the signal. The resulting signals are termed the details (denoted $\mathbb{D}$ ) and approximations (denoted $\mathbb{A}$ ) for the high and low frequency components respectively. Each subsequent $i$ level of DWT applies the same process to $\mathbb{A}[i-1]$, i.e., the approximations at the previous level, as illustrated in Figure 18. We favour DWT throughout this paper when referring to wavelet analysis. 